\title{
POLYNEUTRON THEORY OF TRANSMUTATION
}

\author{
JOHN C. FISHER \\ 600 Arbol Verde, Carpinteria, CA 93013, USA \\ E-mail:jcfisher@fisherstone.com
}

\begin{abstract}
Polyneutron theory is applied to nuclear transmutation. Implications of the theory are compared with experiment. Additional more definitive experiments are suggested.
\end{abstract}

Polyneutron theory postulates that large clusters of neutrons are bound and stable against strong decay and that their interactions with ordinary nuclei are responsible for a new class of low-temperature nuclear phenomena. It is postulated that these clusters, also termed polyneutrons or neutron isotopes, grow to include hundreds of neutrons in chain reactions fueled by isotopes such as ${ }^{2} \mathrm{H},{ }^{18} \mathrm{O}$, and ${ }^{7} \mathrm{Li}^{1}{ }^{1}$ It is anticipated that an ordinary nucleus ${ }_{z}^{A} \mathrm{X}$ and a polyneutron ${ }^{\mathrm{B}} \mathrm{n}$ can bind to form a composite ${ }_{z}^{A} \mathrm{X}^{\mathrm{B}} \mathrm{n}$ that subsequently decays by transfers or exchanges of nucleons between its components. The present analysis focuses on composite formation and on the transmutations that result from composite decay.

I assume that the neutrons in polyneutrons are paired with the BCS symmetry first described for electrons in superconductors, ${ }^{2}$ and that breaking a pair requires so much energy that odd-numbered polyneutrons can be produced only in reaction with deuterium. In the reaction ${ }^{2 A} \mathrm{n}+{ }^{2} \mathrm{H} \rightarrow{ }^{2 A+1} \mathrm{n}+{ }^{1} \mathrm{H}$ transmutation of $\mathrm{H}$ makes available up to $5.847 \mathrm{MeV}$ for adding the odd neutron. If the mass excess of ${ }^{2 A+1} \mathrm{n}$ were to exceed that of ${ }^{2 A} \mathrm{n}$ by (say) $5 \mathrm{MeV}$, the reaction forming it would be exothermic by $0.847 \mathrm{MeV}$. On the other hand the reaction ${ }^{2 A+1} \mathrm{n}+{ }^{2} \mathrm{H} \rightarrow{ }^{2 A+2} \mathrm{n}+{ }^{1} \mathrm{H}$ would be exothermic by $10.847 \mathrm{MeV}$ with a much larger cross section, and in a chain reaction where polyneutrons are growing and fissioning in interaction with ${ }^{2} \mathrm{H}$, as in the experiments of Iwamura et al., ${ }^{3-5}$ the concentration of even polyneutrons is expected to substantially exceed that of odd ones. Hence as a first approximation I consider only even polyneutrons.

Transmutations associated with composite formation and decay are expected to occur at differing rates. Formation is expected to be the limiting rate, depending as it does on the very small concentration of polyneutrons. Strong reactions that do not require associated beta decay or electron capture are assumed to be most rapid, and to occur during composite formation. Weak reactions that require associated beta decay or electron capture are assumed to be slower. Weak reactions that require associated double beta decay or double electron capture are assumed to be slower still, followed by reactions that require three or more associated weak 
reactions. With the foregoing assumptions the following procedure determines the sequence of transmutations according to the present status of the theory:

Step 1. Consider a starting element ${ }_{z}^{\mathrm{C}} \mathrm{X}$ for which we desire to know the isotopes to which it may be transmuted by interaction with polyneutrons. The first step is formation of a composite ${ }_{z}^{A} \mathrm{X}^{\mathrm{B}} \mathrm{n}$ in a reaction such as ${ }_{z}^{\mathrm{C}} \mathrm{X}+{ }^{\mathrm{D}} \mathrm{n} \rightarrow{ }_{z}^{A} \mathrm{X}^{\mathrm{B}} \mathrm{n}+$ $\mathrm{C}+\mathrm{D}-A-\mathrm{B} n$. Such a reaction can only occur in an environment where polyneutrons are continuously being created, as in the active region of an ongoing chain reaction. In a newly formed composite the value of $A$ is that for which the composite is stable against the strong reactions ${ }_{z}^{A} \mathrm{X}^{\mathrm{B}} \mathrm{n} \rightarrow{ }^{A+N}{ }_{z} \mathrm{X}^{\mathrm{B}-N} \mathrm{n}$ for all positive and negative even values of $N$. These reactions must be endothermic. The energy they release is $E_{0}=\Delta\left({ }_{z}^{A} \mathrm{X}\right)-\Delta\left({ }_{z}^{A+N} \mathrm{X}\right)+N \delta$, where $\delta$ represents half the change in polyneutron mass excess associated with adding a pair of neutrons, $\delta=(1 / 2)\left[\Delta\left({ }^{A+2} \mathrm{n}\right)-\Delta\left({ }^{A} \mathrm{n}\right)\right]$. The reaction is exothermic for $E_{0}>0$. Are any such transfers exothermic? If yes, choose the most exothermic among $N= \pm 2, \pm 4, \pm 6, \ldots$, and go to Step 2. If no, go directly to Step 2 .

Step 2. Represent the output of the previous step by ${ }_{z}^{A} \mathrm{X}$. Is there an isotope ${ }_{z \pm 1}^{A+N} \mathrm{Y}$ of the element having one more or one less proton than ${ }_{z}^{A} \mathrm{X}$ that can be reached by exchange or transfer of nucleons within the composite? (Each of these reactions requires an associated beta decay or electron capture.) The energy released is $E_{1}=\Delta\left({ }_{z}^{A} \mathrm{X}\right)-\Delta\left({ }_{z \pm 1}^{A+N} \mathrm{Y}\right)+N \delta$. Are any such transfers exothermic? If yes, choose the most exothermic among $z= \pm 1$ and $N=0, \pm 2, \pm 4, \ldots$, and return to Step 2 . If no, go to Step 3.

Step 3. Represent the output of the previous step by ${ }_{z}^{A} \mathrm{X}$. Is there an isotope ${ }_{z \pm 2}^{A+N} \mathrm{Y}$ of the element having two more or two fewer protons than ${ }_{z}^{A} \mathrm{X}$ that can be reached by exchange or transfer of nucleons within the composite? (Each of these transfers requires two associated beta decays or electron captures.) The energy released is $E_{2}=\Delta\left({ }_{z}^{A} \mathrm{X}\right)-\Delta\left({ }_{z \pm 2}^{A+N} \mathrm{Y}\right)+N \delta$. Are any such transfers exothermic? If yes, choose the most exothermic among $z \pm 1$ and $N=0, \pm 2, \pm 4, \ldots$, and go to Step 2. If no, continue with three or more associated weak decays, or stop if transmutations with such decays are neglected.

The beta decays in Steps 2 and 3 can be imagined as occurring in the polyneutron in association with transfer of the resulting proton to the ordinary nucleus. For $N=0$ each proton so formed exchanges with a neutron from the ordinary nucleus, accelerating the rate of $N=0$ beta decays to match that of $N \neq 0$ decays. Electron captures can be imagined as occurring in association with transfer of a proton from the ordinary nucleus to the polyneutron. For $N=0$ each proton so transferred exchanges with a neutron from the polyneutron, accelerating the rate of $N=0$ electron captures to match that of $N \neq 0$ captures.

Examples of reactions and their energies are shown here in more detail.

No beta decay:

$$
\begin{gathered}
{ }_{z}^{A} \mathrm{X}^{\mathrm{B}} \mathrm{n} \rightarrow{ }_{z}^{A+N} \mathrm{X}^{\mathrm{B}-N} \mathrm{n}+E_{0}, \\
E_{0}=\Delta\left({ }_{z}^{A} \mathrm{X}\right)-\Delta\left({ }_{z}^{A+N} \mathrm{X}\right)+N \delta .
\end{gathered}
$$


Single beta decay:

$$
\begin{gathered}
{ }_{z}^{A} \mathrm{X}^{\mathrm{B}} \mathrm{n} \rightarrow{ }_{z+1}^{A+N} \mathrm{Y}^{\mathrm{B}-N} \mathrm{n}+E_{1}, \\
E_{1}=\Delta\left({ }_{z}^{A} \mathrm{X}\right)-\Delta\left({ }_{z+1}^{A+N} \mathrm{Y}\right)+N \delta .
\end{gathered}
$$

Single electron capture:

$$
\begin{gathered}
{ }_{z}^{A} \mathrm{X}^{\mathrm{B}} \mathrm{n} \rightarrow{ }_{z-1}^{A+N} \mathrm{Y}^{\mathrm{B}-N} \mathrm{n}+E_{1}, \\
E_{1}=\Delta\left({ }_{z}^{A} \mathrm{X}\right)-\Delta\left({ }_{z-1}^{A+N} \mathrm{Y}\right)+N \delta .
\end{gathered}
$$

Double beta decay:

$$
\begin{gathered}
{ }_{z}^{A} \mathrm{X}^{\mathrm{B}} \mathrm{n} \rightarrow{ }_{z+2}^{A+N} \mathrm{Y}^{\mathrm{B}-N} \mathrm{n}+E_{2}, \\
E_{2}=\Delta\left({ }_{z}^{A} \mathrm{X}\right)-\Delta\left({ }_{z+2}^{A+N} \mathrm{Y}\right)+N \delta .
\end{gathered}
$$

Double electron capture:

$$
\begin{gathered}
{ }_{z}^{A} \mathrm{X}^{\mathrm{B}} \mathrm{n} \rightarrow{ }_{z-2}^{A+N} \mathrm{Y}^{\mathrm{B}-N} \mathrm{n}+E_{2}, \\
E_{2}=\Delta\left({ }_{z}^{A} \mathrm{X}\right)-\Delta\left({ }_{z-2}^{A+N} \mathrm{Y}\right)+N \delta .
\end{gathered}
$$

In my calculations, I assume that $\delta$ is independent of $A$ with the value $\delta=1.143$ as tentatively determined from transmutation of ${ }^{138} \mathrm{Ba}$ to ${ }^{150} \mathrm{Sm}$. With this value of $\delta$, and assuming that the binding energy of a composite is independent of the properties of its components, it is possible to calculate the transmutation chain for any starting isotope. (The assumption of constant $\delta$ holds only approximately over a limited range of values of $A$, and the assumption of a constant binding energy for composites ignores the influence of shell structure of the ordinary nucleus and of the sizes of both components. These questions should be addressed in a more realistic treatment of the theory.)

Transmutation chains can be more easily visualized by simplifying the notation. Abbreviate the composite ${ }^{A} \mathrm{X}^{\mathrm{B}} \mathrm{n}$ as $\left({ }^{A} \mathrm{X}\right)$ where the size of the polyneutron component is understood. Then as an example the transmutation ${ }^{139} \mathrm{La}^{\mathrm{B}} \mathrm{n} \rightarrow{ }^{141} \mathrm{Ce}^{\mathrm{B}-2} \mathrm{n}$ is abbreviated $\left({ }^{139} \mathrm{La}\right) \rightarrow\left({ }^{141} \mathrm{Ce}\right)$. With this notation, and neglecting transmutations with three or more associated weak interactions, the transmutation chain for ${ }^{139} \mathrm{La}$ is

$$
{ }^{139} \mathrm{La}+{ }^{A} \mathrm{n} \rightarrow\left({ }^{139} \mathrm{La}\right) \rightarrow\left({ }^{141} \mathrm{Ce}\right) \rightarrow\left({ }^{141} \mathrm{Pr}\right) \rightarrow\left({ }^{143} \mathrm{Nd}\right) .
$$

In addition to these decay channels, each composite has a side channel that frees the ordinary nucleus and substitutes a helium nucleus, typified by

$$
{ }^{141} \mathrm{Ce}^{A} \mathrm{n} \rightarrow{ }^{4} \mathrm{He}^{A-4} \mathrm{n}+{ }^{141} \mathrm{Ce} .
$$

In consequence, accompanying the composite decay chain there is in its wake a residual of free isotopes ${ }^{141} \mathrm{Ce},{ }^{141} \mathrm{Pr},{ }^{143} \mathrm{Nb}$ whose numbers depend on the relative magnitudes of the side channels. To recapitulate, I assume that formation of the initial composite $\left({ }^{139} \mathrm{La}\right)$ is the rate-limiting step. Once a composite has formed, I assume that it decays rapidly through the chain leaving behind a residue of free 
transmuted isotopes that can be revealed by mass spectrometry. Shortly after composite formation ceases, signals for ${ }^{139} \mathrm{La},{ }^{141} \mathrm{Ce},{ }^{141} \mathrm{Pr}$, and ${ }^{143} \mathrm{Nb}$ would be revealed; perhaps with the largest signal for ${ }^{143} \mathrm{Nb}$ if the side reactions are small and most composites survive to the end, or perhaps with the smallest signal if the side reactions are larger and deplete the composites as they pass through successive transmutations. At a later time after composite formation ceases mass spectrometry would detect signals only for ${ }^{139} \mathrm{La},{ }^{141} \mathrm{Pr}$, and ${ }^{143} \mathrm{Nb}$ with an enhanced ${ }^{141} \mathrm{Pr}$ signal from beta decay of ${ }^{141} \mathrm{Ce}$ with its 33 day half life.

I now consider the transmutations investigated by Iwamura et $a l^{3-5}$ Transmutation of ${ }^{138} \mathrm{Ba}$ is reported to lead to ${ }^{150} \mathrm{Sm}$. The decay chain predicted by polyneutron theory is

$$
{ }^{138} \mathrm{Ba}+{ }^{A} \mathrm{n} \rightarrow\left({ }^{138} \mathrm{Ba}\right) \rightarrow\left({ }^{140} \mathrm{Ce}\right) \rightarrow\left({ }^{144} \mathrm{Nd}\right) \rightarrow\left({ }^{150} \mathrm{Sm}\right) \rightarrow\left({ }^{158} \mathrm{Gd}\right) \rightarrow \cdots
$$

leaving residual amounts of ${ }^{140} \mathrm{Ce},{ }^{144} \mathrm{Nd},{ }^{150} \mathrm{Sm},{ }^{158} \mathrm{Gd}$, and so on. It leaves a residue of free ${ }^{150} \mathrm{Sm}$ nuclei only for values of $\delta$ that lie in the narrow range $1.142<\delta<1.144$. This is the basis for my assumption that $\delta=1.143$.

Transmutation of ${ }^{88} \mathrm{Sr}$ is reported to lead to ${ }^{96} \mathrm{Mo}$. The reaction chain predicted by the theory is

$$
{ }^{88} \mathrm{Sr}+{ }^{A} \mathrm{n} \rightarrow\left({ }^{90} \mathrm{Sr}\right) \rightarrow\left({ }^{92} \mathrm{Y}\right) \rightarrow\left({ }^{96} \mathrm{Zr}\right) \rightarrow\left({ }^{96} \mathrm{Nb}\right) \rightarrow\left({ }^{100} \mathrm{Mo}\right) \rightarrow\left({ }^{102} \mathrm{Tc}\right) \rightarrow \cdots
$$

leaving residual amounts of ${ }^{90} \mathrm{Sr},{ }^{92} \mathrm{Y},{ }^{96} \mathrm{Zr},{ }^{96} \mathrm{Nb},{ }^{100} \mathrm{Mo},{ }^{102} \mathrm{Tc}$, and so on. The ${ }^{96} \mathrm{Nb}$ residue decays to ${ }^{96} \mathrm{Mo}$ with a half life of $23 \mathrm{~h}$. During composite formation, mass spectrometry will reveal a signal at mass 96 from residual ${ }^{96} \mathrm{Zr}$ and ${ }^{96} \mathrm{Nb}$, along with ${ }^{96} \mathrm{Mo}$ from decay of ${ }^{96} \mathrm{Nb}$. A few days after composite formation has ceased the mass signal will come from ${ }^{96} \mathrm{Zr}$ and ${ }^{96} \mathrm{Mo}$ with an enhanced ${ }^{96}$ Mo component from decay of ${ }^{96} \mathrm{Nb}$. A Mo signal from XPS will be obtained from the ${ }^{96} \mathrm{Mo}$ and ${ }^{100}$ Mo residuals.

Transmutation of ${ }^{133} \mathrm{Cs}$ is reported to lead to ${ }^{141} \mathrm{Pr}$, and to as yet unidentified isotopes with intermediate masses between 133 and 141. The theory predicts

$$
{ }^{133} \mathrm{Cs}+{ }^{A} \mathrm{n} \rightarrow\left({ }^{137} \mathrm{Cs}\right) \rightarrow\left({ }^{137} \mathrm{Ba}\right) \rightarrow\left({ }^{139} \mathrm{La}\right) \rightarrow\left({ }^{141} \mathrm{Ce}\right) \rightarrow\left({ }^{141} \mathrm{Pr}\right) \rightarrow\left({ }^{143} \mathrm{Nd}\right)
$$

leaving residual amounts of ${ }^{137} \mathrm{Cs},{ }^{137} \mathrm{Ba},{ }^{139} \mathrm{La},{ }^{141} \mathrm{Ce},{ }^{141} \mathrm{Pr}$, and ${ }^{143} \mathrm{Nd}$. The latter portion of this chain was treated in the example above for transmutation of ${ }^{139} \mathrm{La}$, in which a signal for ${ }^{141} \mathrm{Pr}$ arose from the ${ }^{141} \mathrm{Pr}$ residual plus additional ${ }^{141} \mathrm{Pr}$ from decay of ${ }^{141} \mathrm{Ce}$.

Finally transmutation of ${ }^{137} \mathrm{Ba}$ was reported to lead to a signal at mass 149 , tentatively attributed to ${ }^{149} \mathrm{Sm}$ although no XPS signal for Sm could be detected. Theory predicts the decay chain

$$
{ }^{137} \mathrm{Ba}+{ }^{A} \mathrm{n} \rightarrow\left({ }^{137} \mathrm{Ba}\right) \rightarrow\left({ }^{139} \mathrm{La}\right) \rightarrow\left({ }^{141} \mathrm{Ce}\right) \rightarrow\left({ }^{141} \mathrm{Pr}\right) \rightarrow\left({ }^{143} \mathrm{Nd}\right)
$$

leaving residual amounts of ${ }^{139} \mathrm{La},{ }^{141} \mathrm{Ce},{ }^{141} \mathrm{Pr}$, and ${ }^{143} \mathrm{Nd}$ as for transmutation of ${ }^{133} \mathrm{Cs}$. No signal for $\mathrm{Sm}$ is expected. (It may be that the reported mass 149 signal represents ${ }^{137} \mathrm{Ba}^{12} \mathrm{C}$ or ${ }^{133} \mathrm{Cs}^{16} \mathrm{O}$ from contamination by carbon or cesium during the transmutation process.) 
Overall the agreement between theory and the experiments of Iwamura et al. is suggestive but not definitive. Consideration of other transmutation chains identifies experiments that could be more definitive. The parameter choice $\delta=1.143$ implies that ${ }^{207} \mathrm{~Pb}$ and ${ }^{208} \mathrm{~Pb}$ are absolutely stable, all potential transmutations involving any number of possible associated weak interactions being endothermic. Looked at another way, these $\mathrm{Pb}$ isotopes are the final products of transmutation of every starting element, provided only that sufficient time is allowed. Nuclei lighter than lead transmute by beta decay alone, as in

$$
{ }^{205} \mathrm{Tl}+{ }^{A} \mathrm{n} \rightarrow\left({ }^{205} \mathrm{Tl}\right) \rightarrow\left({ }^{207} \mathrm{~Pb}\right) .
$$

Nuclei heavier than lead transmute by electron capture alone, as in

$$
{ }^{209} \mathrm{Bi}+{ }^{A} \mathrm{n} \rightarrow\left({ }^{209} \mathrm{Bi}\right) \rightarrow\left({ }^{207} \mathrm{~Pb}\right) .
$$

Lead isotopes ${ }^{204} \mathrm{~Pb}$ and ${ }^{206} \mathrm{~Pb}$ transmute by neutron transfer in association with composite formation, as in

$$
\begin{aligned}
& { }^{204} \mathrm{~Pb}+{ }^{A} \mathrm{n} \rightarrow\left({ }^{208} \mathrm{~Pb}\right), \\
& { }^{206} \mathrm{~Pb}+{ }^{A} \mathrm{n} \rightarrow\left({ }^{208} \mathrm{~Pb}\right) .
\end{aligned}
$$

These transmutations are ideal testing grounds for the theory. They suggest that polyneutrons can induce transmutations where the final nucleus differs from the starting nucleus by addition of a proton and a neutron, by subtraction of a proton and a neutron, or by addition of two or four neutrons. Each of these reactions requires only a single step from composite formation to the final ${ }^{207} \mathrm{~Pb}$ or ${ }^{208} \mathrm{~Pb}$ product. Because the product is stable, one-to-one substitution of ${ }^{207} \mathrm{~Pb}$ for ${ }^{205} \mathrm{Tl}$ and for ${ }^{209} \mathrm{Bi}$ should be seen, as should substitution of ${ }^{208} \mathrm{~Pb}$ for ${ }^{204} \mathrm{~Pb}$ and ${ }^{206} \mathrm{~Pb}$.

The predictions of the theory are definite and clear-cut, although minor corrections can be expected when the analysis is extended to include odd polyneutrons. If the predictions are borne out by experiment the credibility of the theory will be enhanced. If not the theory will face a crisis.

\section{References}

1. J. C. Fisher, Fusion Tech. 34, 66 (1998).

2. J. Bardeen, L. N. Cooper, and J. R. Schrieffer, Phys. Rev. 108, 1175 (1957).

3. Y. Iwamura, M. Sakano, and T. Itoh, Jpn. J. Appl. Phys., 41, 4642 (2002).

4. Y. Iwamura, T. Itoh, M. Sakano, N. Yamazaki, S. Kuribayashi, Y. Terada, T. Ishikawa, and J. Kasagi, Proc. ICCF 11 (World Scientific Inc., 2006), 339.

5. Y. Iwamura, T. Itoh, M. Sakano, N. Yamazaki, S. Kuribayashi, Y. Terada, and T. Ishikawa, Proc. ICCF 12 (World Scientific Inc., 2006), to be published. 